\title{
Diversity of Nontuberculous Mycobacteria in Kuwait: Rapid Identification and Differentiation of Mycobacterium Species by Multiplex PCR, INNO-LiPA Mycobacteria v2 Assay and PCR Sequencing of rDNA
}

\author{
Suhail Ahmad Eiman Mokaddas \\ Department of Microbiology, Faculty of Medicine, Kuwait University, Safat, Kuwait
}

\section{Significance of the Study}

- Specific identification of Mycobacterium tuberculosis (MTB) and nontuberculous mycobacteria (NTM) is crucial for appropriate treatment of mycobacterial infections. Multiplex PCR differentiated MTB from NTM. Line probe assay (LiPA) and/or PCR sequencing studies identified 15 NTM species/subspecies among 53 NTM isolates. Rapid MTB/NTM differentiation by PCR followed by NTM identification by LiPA/PCR sequencing is suitable for proper management of mycobacterial infections in Kuwait.

\section{Keywords}

Mycobacterial identification · NTM species · Multiplex PCR · INNO LiPA Mycobacteria v2 assay · PCR sequencing of 16S-23S rDNA

\begin{abstract}
Objective: Nontuberculous mycobacteria (NTM) often cause disease that is clinically indistinguishable from tuberculosis. Specific identification is important as treatment varies according to Mycobacterium species causing the infection. This study used multiplex PCR (MPCR) assay for rapid differentiation of mycobacterial growth indicator tube 960 system (MGIT) cultures as Mycobacterium tuberculosis (MTB) or NTM together with INNO LiPA Mycobacteria v2 assay (LiPA) and/ or PCR sequencing of rDNA for species-specific identification of selected MTB and all NTM isolates in Kuwait. Materials
\end{abstract}

\begin{tabular}{ll}
\hline KARGER & $\begin{array}{l}\text { ( ) 2019 The Author(s) } \\
\text { Published by S. Karger AG, Basel Openger }\end{array}$ \\
E-Mail karger@karger.com & $\begin{array}{l}\text { This is an Open Access article licensed under the Creative Commons } \\
\text { Attribution-NonCommercial-4.0 International License (CC BY-NC) } \\
\text { (http://www.karger.com/Services/OpenAccessLicense), applicable to } \\
\text { the online version of the article only. Usage and distribution for com- } \\
\text { mercial purposes requires written permission. }\end{array}$
\end{tabular}

and Methods: DNA was extracted from MGIT cultures ( $n=$ 1,033) grown from 664 pulmonary and 369 extrapulmonary specimens from 1,033 suspected tuberculosis patients. mPCR was performed to differentiate MTB from NTM. LiPA was performed and results were interpreted according to kit instructions. rDNA was amplified and sequenced by using panmycobacterial primers. Results: $\mathrm{mPCR}$ identified 979 isolates as MTB, 53 as NTM and 1 isolate as mixed culture. LiPA and/or PCR sequencing confirmed 112 of 979 selected isolates as MTB. Mixed culture contained $M$. tuberculosis and $M$. fortuitum. LiPA yielded 12 patterns and identified 10 species/ species complexes among 47 NTM, M. kansasii + M. scrofulaceum in one culture and 5 isolates only at genus level. PCR sequencing yielded more specific identification for 22 isolates at the species/subspecies level. Conclusions: $\mathrm{mPCR}$ rapidly differentiated MTB from NTM. LiPA identified 44 of 52 NTM isolates at the species/species complex level and 2 
mixed cultures. PCR sequencing yielded more specific identification at the species/subspecies level. Rapid differentiation as MTB or NTM by MPCR, followed by species-specific NTM identification by LiPA/PCR sequencing is suitable for the proper management of mycobacterial infections in Kuwait.

(c) 2019 The Author(s)

Published by S. Karger AG, Basel

\section{Introduction}

The genus Mycobacterium contains more than 140 species which belong either to the Mycobacterium tuberculosis (MTB) complex, obligate human/animal pathogens, or to numerous nontuberculous mycobacteria (NTM). The NTM are a diverse group of ubiquitous environmental opportunistic human pathogens frequently isolated from water, soil, dust, and plants $[1,2]$. Although tuberculosis (TB) is the most common mycobacterial infection in developing countries, the incidence of NTM infections is increasing rapidly, and these infections now surpass TB in resource-rich developed countries $[3,4]$. Potential contributing factors for increase in NTM infections include an increase in the population of susceptible hosts such as elderly subjects and individuals with defects in the cellular immune system due to disease or immunosuppressive treatment $[2,4]$. The infection is mainly acquired as a result of contact with contaminated environments via inhalation, ingestion or dermal contact, and results in lymphadenitis or pulmonary, disseminated and skin/soft tissue infection while human-to-human transmission is usually excluded $[2,4,5]$. Pulmonary NTM infections are most frequent and some NTMs such as Mycobacterium kansasii and Mycobacterium abscessus can also cause lung or other infections in apparently healthy subjects; however, infections involving extrapulmonary body sites are also increasing [2, 4-7]. NTM often cause disease that is clinically and radiographically indistinguishable from TB; however, treatment strategies and the prognosis of NTM disease are quite different $[2,5-8]$.

Different species of NTM are divided nearly equally between rapid growers showing visible growth on solid media within 7 days and slow growers requiring incubation times of several weeks, similar to M. tuberculosis. The two groups of NTM also differ in their susceptibility to antimicrobials, preferential disease localization, and prognosis $[2,5]$. Thus, specific identification of NTM is also crucial for appropriate treatment of mycobacterial infections. Most epidemiological studies of NTM have been performed in developed countries including Japan, Australia, and countries in North America and Europe $[4-7,9]$. The epidemiology of NTM infections from Saudi Arabia has recently shown highly diverse diseases involving common and rare species of NTM [10-12]; however, only scant information is available from Kuwait and other Arabian Gulf countries [13, 14]. In Kuwait, nearly 800 mycobacterial infections are diagnosed every year [15, E. Mokaddas, unpublished data]. Nearly $95 \%$ of mycobacterial infections in Kuwait are caused by M. tuberculosis (infections by other members of $M$. tuberculosis complex are extremely rare) while the remaining $~ 5 \%$ are caused by NTM $[13,15]$. Nearly $80 \%$ of all TB cases and nearly $90 \%$ of drug-resistant TB cases in Kuwait occur in expatriate individuals mainly originating from TB-endemic countries of South/South East Asia [15]. On the contrary, NTM infections were more common among Kuwaiti nationals, and only 7 NTM species were identified in a small epidemiological study carried out previously [13]. This large-scale study was, therefore, carried out to detect NTM species among all available mycobacterial growth indicator tube 960 system (MGIT) cultures obtained from pulmonary and extrapulmonary specimens from patients with presumptive TB during a 2-year period in Kuwait. A multiplex PCR (mPCR) assay was used first for rapid differentiation of MGIT cultures as MTB or NTM. Further species-specific identification of NTM was achieved by INNO LiPA Mycobacteria v2 assay (LiPA) and/or PCR sequencing of 16S-23S internally transcribed spacer (ITS) region of rDNA.

\section{Materials and Methods}

Reference Strains and Clinical Mycobacterium Species Isolates

Reference strains of MTB $\left(\mathrm{H}_{37} \mathrm{Rv}\right)$ and NTM were used to validate the results of LiPA and for comparing PCR sequencing data for the ITS region of rDNA. We used a total of 1,033 Mycobacterium species isolates (representing all available isolates obtained during a 2-year period from suspected TB patients referred to $\mathrm{Ku}$ wait National TB Control Laboratory) available from heat-killed culture collection. Clinical specimens were collected after obtaining consent from suspected TB patients as part of routine diagnostic workup. The isolates were grown from 664 pulmonary and 369 extrapulmonary specimens collected from 1,033 patients with presumptive TB after obtaining verbal consent as part of routine patient care. Nonsterile clinical specimens were processed by the standard N-acetyl-L-cysteine and sodium hydroxide (NALC/ $\mathrm{NaOH}$ ) method while sterile samples were processed directly for culture in automated MGIT (Beckton-Dickinson, Sparks, MD, USA) according to the manufacturer's instructions and as described previously $[15,16]$. Data analyses were carried out without revealing patient identity. 
Fig. 1. Representative agarose gel of $\mathrm{mP}$ CR-amplified products using genomic DNA extracted from mycobacterial growth indicator tube 960 system (MGIT) cultures of 9 selected Mycobacterium species and showing an MTB-specific pattern (lanes $1-4,6,8)$ or an NTM-specific pattern (lanes 5,7 ) or a mixed MTB + NTM pattern (lane 9). Lane $\mathrm{M}$ is $100 \mathrm{bp}$ DNA ladder and the position of migration of 100-, 300-, and 600-bp fragments are marked.

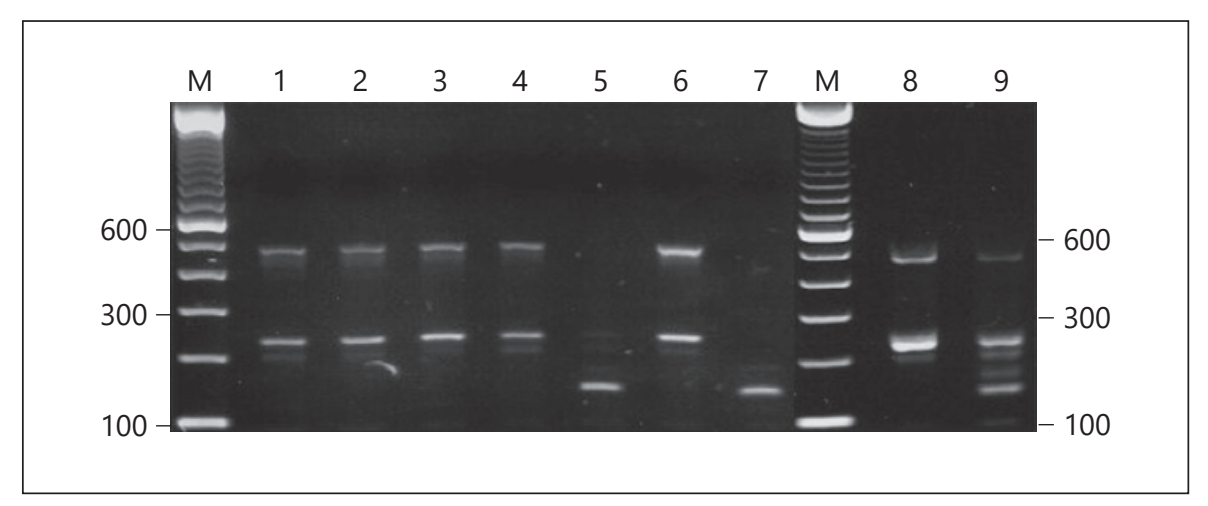

Template DNA Preparation and mPCR Assay

Genomic DNA was prepared from heat-killed MGIT cultures of Mycobacterium species isolates by incorporating the removal of PCR inhibitors with Chelex-100 (Sigma-Aldrich Co., St. Louis, MO, USA) as described previously [17]. The isolates were initially subjected to mPCR assay, performed as described previously [18], which targets the $o x y R$-ahpC intergenic region and variable region of $r p o B$ gene sequences and identifies Mycobacterium species isolates as MTB or NTM based on pattern and sizes of amplified fragments.

INNO LiPA Mycobacteria v2 Line Probe Assay (LiPA)

Species-specific identification of selected MTB and all NTM isolates was carried out by using a commercial reverse hybridization-based line probe assay with the INNO-LiPA Mycobacteria v2 (LiPA) kit (Fujeribio, Ghent, Belgium). In brief, PCR amplification was performed with biotinylated primers targeting the $16 \mathrm{~S}-23 \mathrm{~S}$ ITS region of rDNA, and the amplicons were denatured and hybridized with 23 oligonucleotide probes immobilized on membrane strips. The captured amplified DNA was detected, and the results were interpreted according to the instructions supplied with the kit. Water was used instead of DNA for negative controls (blank tube) with each run of LiPA.

PCR Sequencing of the 16S-23S ITS Region of rDNA

Species-specific identification of some selected MTB and all NTM isolates was also carried out by PCR amplification followed by DNA sequencing (PCR sequencing) of the 16S-23S ITS region of rDNA. The ITS region of rDNA was amplified by using panmycobacterial forward (SAKW135; 5'-GATTGGGACGAAGTCGTAACAAG-3') and reverse (SAKW136, 5'-AGCCTCCCACGTCCTTCATCGGCT-3') primers and the touchdown PCR amplification and cycling conditions described previously [17]. The amplicons were purified by using a PCR product purification kit (Qiagen, Hilden, Germany). Both strands of purified amplicons were sequenced by using the same amplification primers as sequencing primers and $\mathrm{ABI}$ BigDye terminator (version 3.1) cycle sequencing kit (Life Technologies Corp., Austin, TX, USA). Sequencing reactions were performed and processed as described previously [19].

Sequence data were checked for confidence levels with an ABI sequence scanner, reverse complements were generated and aligned with forward sequences using Clustal Omega (http://www. ebi.ac.uk/Tools/msa/clustalo/). Basic local alignment search tool
(BLAST) searches (http://www.ncbi.nlm.nih.gov/BLAST/Blast. cgi) of DNA sequence data were performed with data from GenBank and sequence identity of $>99 \%$ with reference strains or wellcharacterized clinical isolates of various Mycobacterium species was used for species identification.

\section{Results}

A total of 1,033 unique Mycobacterium species isolates collected from 1,033 patients who had presented to the Kuwait National TB Control Laboratory with presumed TB (representing all available Mycobacterium species isolates obtained during a 2-year period) were used in this study. The isolates were cultured from 664 pulmonary and 369 extrapulmonary specimens. Pulmonary specimens included sputum $(n=589)$, bronchoalveolar lavage $(n=62)$, gastric aspirate $(n=12)$, and endotracheal secretion $(n=1)$. Extrapulmonary specimens yielding Mycobacterium species isolates included pus/fine needle aspirate $(n=264)$, lymph node $(n=30)$, tissue biopsy $(n=30)$, pleural fluid ( $n=20)$, cerebrospinal fluid $(n=13)$, urine $(n=6)$, pericardial fluid $(n=3)$, ascitic fluid $(n=2)$, and synovial fluid $(n=1)$. The genomic DNA obtained from 1,033 MGIT cultures flagged positive for bacterial growth were first tested by mPCR to differentiate MTB from NTM species.

The mPCR assay (data from 9 selected isolates are shown in Fig. 1) identified 979 isolates as MTB, 53 isolates as NTM, and 1 isolate as mixed culture containing MTB and an NTM species. The results of mPCR were extended by LiPA and PCR sequencing of the ITS region of rDNA. PCR amplification of genomic DNA with LiPA primers yielded amplicons of nearly 400-550 bp (data from 8 selected isolates are shown in online suppl. Fig. S1; see www.karger.com/doi/10.1159/000498910 for all online suppl. material) from different Mycobacterium species. 
Table 1. Differentiation of MGIT cultures as MTB or NTM by MPCR and species-specific identification of selected MTB and all NTM isolates by INNO LiPA Mycobacteria v2 assay (LiPA) and PCR sequencing of the 16S-23S ITS region

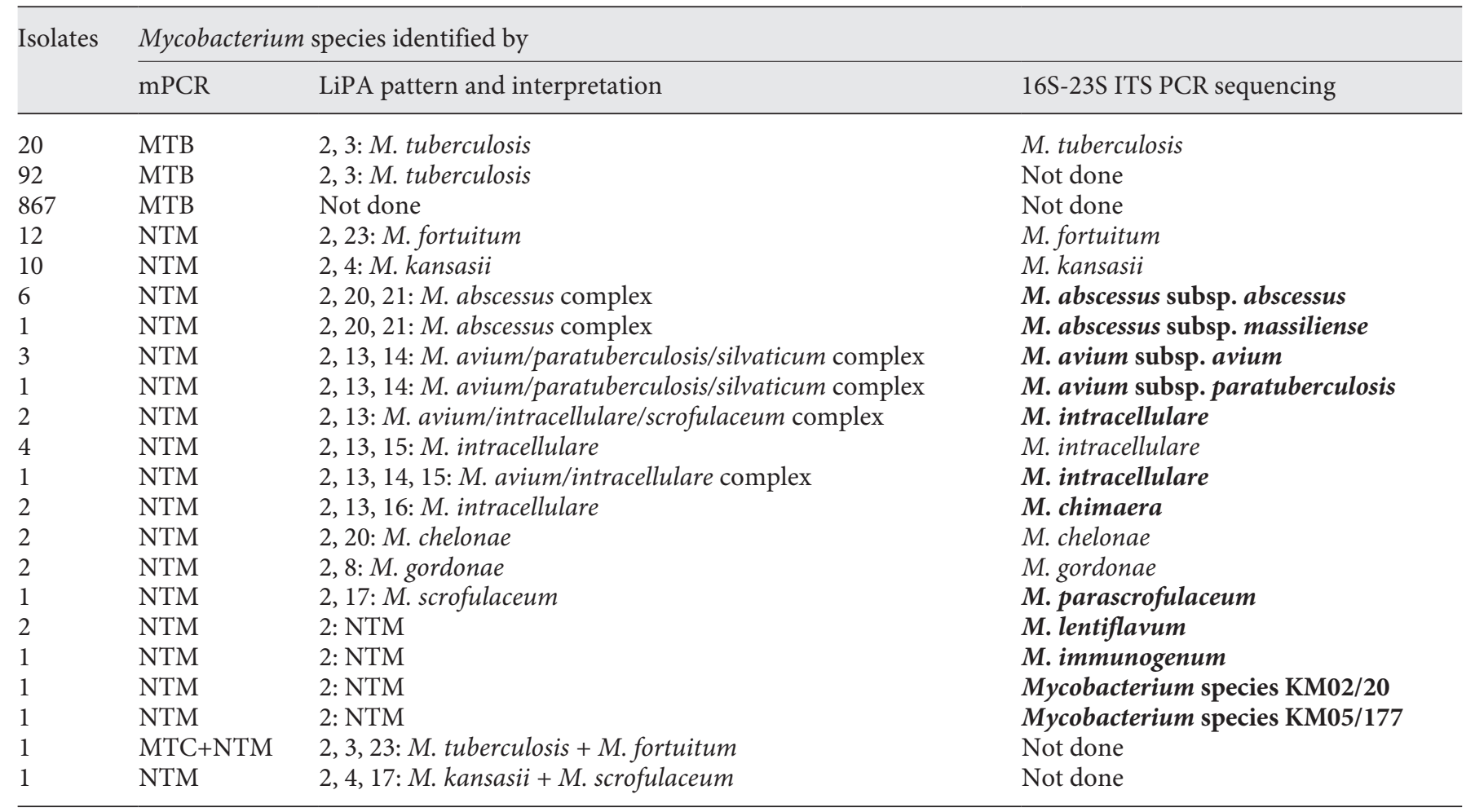

More specific identifications of NTM obtained by PCR sequencing of the 16S-23S ITS region of rDNA are indicated in bold.

When the amplicons were hybridized with probe primers on LiPA strips, interpretable results (data from 13 selected isolates are shown in online suppl. Fig. S2) were obtained from all tested isolates. LiPA identified all 112 randomly selected MTB isolates as M. tuberculosis (Table 1). The ITS region sequences of 20 of 112 isolates were identical and matched completely (100\%) with the corresponding sequence from reference $M$. tuberculosis $\mathrm{H}_{37} \mathrm{Rv}$ (Table 1). The LiPA results also confirmed the presence of MTB and an NTM species in the mixed culture as both M. tuberculosis and Mycobactrium fortuitum were detected simultaneously (Table 1; online suppl. Fig. S2). When LiPA was performed on 53 NTM isolates, 47 isolates were identified at the species/species complex level, 5 isolates were identified only at the genus level (Mycobacterium species), and 1 isolate contained a mixed culture comprising 2 NTM species (Mycobacterium kansasii + Mycobacterium scrofulaceum) (Table 1).

The species-specific identification of NTM by PCR sequencing of the ITS region of rDNA matched completely with LiPA results for 30 isolates belonging to Mycobacte-

Rapid Identification of Mycobacterium

Species in Kuwait rium fortuitum ( $n=12)$, M. kansasii $(n=10)$, Mycobacterium intracellulare $(n=4)$, Mycobacterium chelonae $(n=$ $2)$, and Mycobacterium gordonae $(n=2)$. More specific identification was obtained for 14 NTM isolates and included Mycobacterium abscessus subsp. abscessus $(n=6)$ and M. abscessus subsp. messiliense $(n=1)$ among $7 M y$ cobacterium abscessus complex isolates; Mycobacterium avium subsp. avium $(n=3)$ and M. avium subsp. paratuberculosis ( $n=1)$ among $4 \mathrm{M}$. avium/paratuberculosis/ silvaticum complex isolates; Mycobacterium intracellulare $(n=2)$ among $2 \mathrm{M}$. avium/intracellulare/scrofulaceum complex isolates; and $M$. intracellulare $(n=1)$ in place of $1 \mathrm{M}$. avium/intracellulare complex isolate (Table 1). PCR sequencing also identified 2 isolates detected as $M$. intracellulare by LiPA as Mycobacterium chimaera and 1 isolate detected as M. scrofulaceum by LiPA as M. parascrofulaceum (Table 1). Furthermore, PCR sequencing of 5 NTM isolates identified only at the genus level by LiPA showed that 2 isolates were Mycobacterium lentiflavum, 1 isolate was Mycobacterium immunogenum, 1 isolate was Mycobacterium species KM05-20 and 1 isolate was Myco- 
Table 2. Site of infection and nationality status of 53 patients yielding NTM isolates and species/subspecies-specific identification of all NTM isolates by PCR sequencing of the 16S-23S ITS region of rDNA

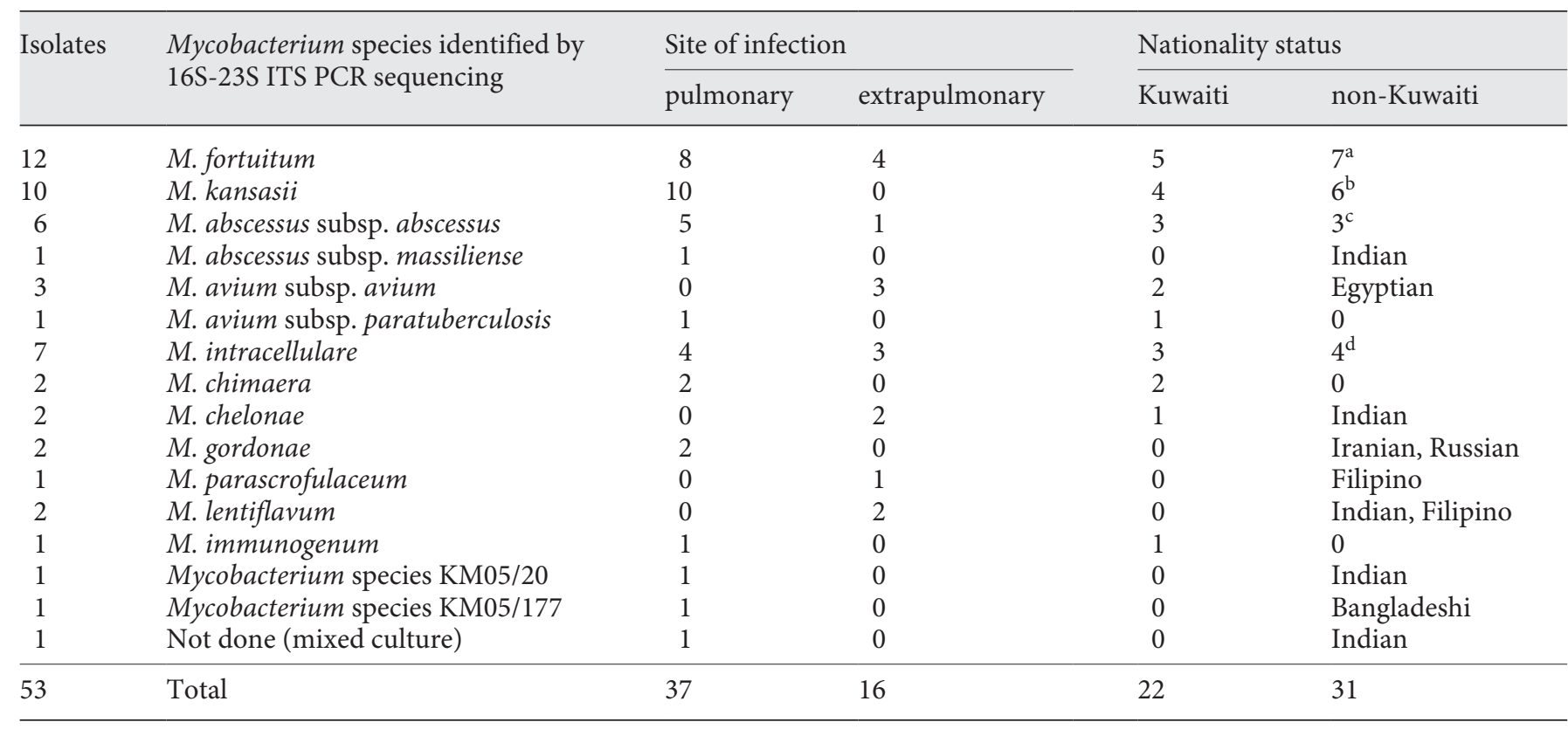

a Indian, 2; Saudi Arabian, 1; Nepali, 1; Pakistani, 1; Bangladeshi, 1; Filipino, $1 .{ }^{\text {b }}$ Bangladeshi, 3; Indian, 1 ; Iranian, 1 ; Australian, 1. ${ }^{\mathrm{c}}$ Indian, 1; Bangladeshi, 1; Filipino, $1 .{ }^{\mathrm{d}}$ Egyptian, 1 ; Indian, 1; Bangladeshi, 1 ; Filipino, 1.

bacterium species KM05-177. PCR sequencing could not be performed for 1 isolate which contained 2 different NTM species (M. kansasii and M. scrofulaceum) and another isolate containing MTB + NTM mixed culture due to technical difficulties.

The clinical specimens and characteristics of patients yielding 53 NTM cultures are presented in Table 2. The NTM isolates were cultured from 37 pulmonary (sputum, 31; bronchoalveolar lavage, 5; endotracheal secretion, 1) and 16 extrapulmonary (pus/fine needle aspirate, 11; urine, 2 ; lymph node, 1; tissue biopsy, 1 ; synovial fluid, 1) samples obtained from 22 Kuwaiti and 31 non-Kuwaiti (Indian, 10; Bangladeshi, 7; Filipino, 5; Iranian, 2; Egyptian, 2; Saudi Arabian, 1; Pakistani, 1; Nepali, 1; Russian, 1; and Australian, 1) patients (Table 2). The NTM were isolated from 37 male and 16 female patients and their age ranged from 3 to 80 years. Only 4 patients were children $<4$ years of age while the remaining 49 patients were adults including 29 patients $\geq 40$ years of age. Interestingly, all M. kansasii isolates were obtained from pulmonary specimens from patients with presumed TB and multiple repeat samples yielding $M$. kansasii were obtained from 8 of 10 patients over a period ranging from few days to nearly 1 month. Most $M$. fortuitum and $M$. abscessus complex isolates were also obtained from pulmonary specimens from patients with presumed TB. Repeat samples yielding M. abscessus subsp. abscessus from 3 patients, $M$. avium subsp. avium from 2 patients and $M$. fortuitum and $M$. intracellulare from 1 patient each were also obtained from patients with presumed TB infection.

The DNA sequence data reported in this study have been submitted to GenBank under accession No. LR031409 to LR031433 and LR031449 to LR031468.

\section{Discussion}

The incidence of NTM infections is increasing worldwide and now surpasses TB in resource-rich developed countries $[4-7,9]$. The distribution of NTM species is not uniform either, as marked geographic variability has been noted both with regard to the prevalence of NTM lung disease and with regard to the NTM species causing these infections $[2,5,12,20]$. The susceptibility of NTM to anti-TB drugs and other antibiotics varies markedly even among members belonging to the same species/species complex which necessitates different treatment strategies for NTM infections and in vitro susceptibility does not 
always correlate with effective in vivo response to antibiotics $[2,5,20]$. Thus, rapid differentiation of MTB from NTM and species-specific identification of NTM is crucial for proper treatment and appropriate patient management.

The application of mPCR on DNA isolated from 1,033 MGIT cultures identified 979 isolates as MTB, 53 isolates as NTM, and 1 isolate as mixed culture containing both MTB and NTM. The identification of 112 selected MTB isolates was confirmed by LiPA and/or PCR sequencing of the ITS region of rDNA. The mixed culture was subsequently shown to contain $M$. tuberculosis $+M$. fortuitum by LiPA. The patient yielding M. tuberculosis $+M$. fortuitum responded to anti-TB treatment suggesting that $M$. fortuitum was most likely a contaminant. Our mPCR data based on a large number of MGIT cultures of $\mathrm{Myco-}$ bacterium species showed that this assay targeting the $o x y R-a h p C$ intergenic region and the variable region of $r p o B$ gene [18] is a rapid, cost-effective and efficient way to differentiate MTB from NTM, and is also capable of identifying mixed MTB + NTM cultures. Other investigators have used more expensive real-time PCR formats with/without expensive probe primers [21,22]. Although matrix-assisted laser desorption ionization-time of flight mass spectrometry (MALDI-TOF MS) has also been used for identification and differentiation of various mycobacteria, the method requires growth on time-consuming solid media for more accurate identification [23, 24].

The LiPA identified 44 NTM isolates to the species/ species complex level and mixed NTM (M. kansasii $+M$. scrofulaceum) infection in 1 isolate. PCR sequencing data yielded more specific identification as 3 isolates ( $M$. chimaera, $n=2$; M. parascrofulaceum, $n=1$ ) were identified as a different species but within the same species complex and 5 isolates identified only at the genus level by LiPA were also identified at the species level (M. lentiflavum, $n=2$; $M$. immunogenum, $n=1$, and 2 novel species, $M y$ cobacterium species KM05/20 and Mycobacterium species KM05/177 not reported previously). Also, 11 isolates (M. abscessus, $n=7 ; M$. avium, $n=4$ ) were identified at the subspecies level. Altogether 11 previously reported and 2 novel species were identified among 53 NTM isolates in Kuwait with M. fortuitum $(n=12)$, M. kansasii $(n=10), M$. abscessus $(n=7), M$. intracellulare $(n=7)$, and $M$. avium $(n=4)$ being the most frequently isolated species. While $M$. avium and $M$. intracellulare are the most commonly isolated NTM in several countries $[5,10$, $20,25,26], M$. fortuitum was also the most common or second most common NTM species in Iran and Saudi Arabia [10, 12, 27]. Interestingly, M. simiae, another

Rapid Identification of Mycobacterium Species in Kuwait common NTM in Saudi Arabia [12], was not detected in Kuwait. The high recovery of $M$. fortuitum from pulmonary specimens $(n=8)$ could be attributed to the use of liquid culture (MGIT) applied for cultivation as it has been shown to enhance the diagnosis of patients with an even milder form of NTM lung disease [26]. However, the significance of isolation of $M$. fortuitum from sputum $(n=8)$ and also possibly from urine $(n=2)$ samples remained unknown as clinical details of the patients yielding these isolates were not available.

The second (M. kansasii) and third (M. abscessus) most commonly isolated NTM in Kuwait are also among the most frequently isolated pulmonary NTM pathogens from several countries including USA, several European countries, Saudi Arabia, South Korea, and China [6, 7, 10, $12,25,26]$. Interestingly, $M$. kansasii and $M$. abscessus are among the relatively more virulent species of NTM which are frequently associated with pulmonary infections, particularly when they are isolated repeatedly from patients with symptoms of pneumonia $[6,7,20,28]$. Consistent with these observations and in line with the criteria laid down by the American Thoracic Society [2], 8 of 10 patients yielded $M$. kansasii from multiple $(\geq 2)$ sputum samples and presented with fever, weight loss, lung cavities and/or hemoptysis. Repeated isolation of M. kansasii from sputum samples is almost always associated with NTM lung disease $[5,6,20]$. Similarly, 3 patients with pulmonary infection also yielded M. abscessus from multiple sputum samples, again suggesting NTM lung disease $[5,6,20,28]$. These observations suggest that most patients yielding $M$. kansasii and M. abscessus in Kuwait had NTM lung disease. Furthermore, prevalence of NTM infections were more common in male and in older $(\geq 40$ years) patients who had presented with presumed TB as has been found in other studies $[6,9,11,12]$.

Our data also showed limited diversity of NTM in Kuwait as only 13 different species were identified including 2 NTM not reported previously. Surprisingly, Mycobacterium riyadhense or other rare NTM species reported recently from neighboring countries [10-12] were not found in Kuwait. Our data also showed that PCR sequencing of the ITS region yielded a more specific identification of NTM at the species/subspecies level which could be clinically useful. For instance, $M$. abscessus sensu lato isolates are divided into at least 3 subspecies: $M$. $a b$ scessus subsp. abscessus, M. abscessus subsp. massiliense, and $M$. abscessus subsp. bolletti $[28,29]$. Antibiotic treatment with clarithromycin is highly effective against lung infections caused by M. abscessus subsp. massiliense while resistance develops more readily during $M$. abscessus sub- 
sp. abscessus lung disease $[7,28,29]$. Interestingly, PCR sequencing identified 1 of 7 M. abscessus complex isolates in Kuwait as M. abscessus subsp. massiliense. PCR sequencing also identified 1 urine isolate as $M$. parascrofulaceum instead of $M$. scrofulaceum (a frequent cause of lymphadenitis) even though clinically both species respond similarly [5] and $2 \mathrm{M}$. intracellulare complex isolates as $M$. chimaera, a less virulent species than $M$. intracellulare, underscoring the importance of correct identification [24]. Although M. chimaera has also been differentiated from $M$. intracellulare by MALDI-TOF MS recently, accurate identification required more rigorous analyses of characteristic peaks in mass spectra [24]. PCR sequencing also identified all 5 isolates detected only at the genus level by LiPA. Two isolates were identified as M. lentiflavum, a recently described NTM species which is a frequent cause of lymphadenitis, particularly in children [5]. Both isolates were obtained from patients with lymphadenitis; however, unlike in other studies [5], both patients were adult expatriates. One isolate obtained from bronchoalveolar lavage of a patient with respiratory symptoms was identified as $M$. immunogenum, another recently described NTM species and a known cause of hypersensitivity pneumonitis [30]. However, additional clinical details including treatment given and the outcome were not available. The remaining 2 isolates were identified as 2 new Mycobacterium species (designated as Mycobacterium sp. KM05-20 and Mycobacterium sp. KM05-177). The ITS region sequences of both these isolates were very different compared to $M$. riyadhense or other common or rare NTM species isolated from neighboring countries [10-12]. Both these species were isolated from sputum samples obtained from 2 expatriate (Indian and Bangladeshi) patients with pulmonary infection, and it is probable that the isolates were acquired from their native countries.
A limitation of our study is that the clinical significance of some NTM isolates, particularly those recovered from sputum and other nonsterile body sites, was not investigated due to lack of information on specific treatments given to the patients and the outcomes.

\section{Conclusion}

Our data on a large collection of MGIT cultures have shown that application of MPCR assay rapidly identified and differentiated 979 MTB from 53 NTM isolates and also identified a mixed culture. LiPA and/or PCR sequencing confirmed identification of all selected isolates as MTB. Mixed culture contained M. tuberculosis and M. fortuitum. LiPA yielded 12 patterns and identified 10 species/species complexes among 47 NTM, M. kansasii $+M$. scrofulaceum in an NTM mixed culture and 5 isolates only at the genus level. PCR sequencing yielded more specific identification at species/subspecies level for 22 isolates which could be important for more appropriate treatment. Thus, rapid differentiation as MTB or NTM by MPCR followed by species-specific NTM identification by LiPA/PCR sequencing is most suitable for proper management of mycobacterial infections in Kuwait.

\section{Acknowledgment}

We thank Nabeel Kunjumoidu for technical assistance.

\section{Disclosure Statement}

The authors have no conflicts of interest to declare.

\section{References}

1 Covert TC, Rodgers MR, Reyes AL, Stelma GN Jr. Occurrence of nontuberculous mycobacteria in environmental samples. Appl Environ Microbiol. 1999 Jun;65(6):2492-6.

2 Griffith DE, Aksamit T, Brown-Elliott BA, Catanzaro A, Daley C, Gordin F, et al; ATS Mycobacterial Diseases Subcommittee; American Thoracic Society; Infectious Disease Society of America. An official ATS/ IDSA statement: diagnosis, treatment, and prevention of nontuberculous mycobacterial diseases. Am J Respir Crit Care Med. 2007 Feb;175(4):367-416.
3 Ahmad S. New approaches in the diagnosis and treatment of latent tuberculosis infection. Respir Res. 2010 Dec;11(1):169.

4 Alcaide F, Peña MJ, Pérez-Risco D, Camprubi D, Gonzalez-Luquero L, Grijota-Camino $\mathrm{MD}$, et al. Increasing isolation of rapidly growing mycobacteria in a low-incidence setting of environmental mycobacteria, 19942015. Eur J Clin Microbiol Infect Dis. 2017 Aug;36(8):1425-32.

5 Tortoli E. Clinical manifestations of nontuberculous mycobacteria infections. Clin Microbiol Infect. 2009 Oct;15(10):906-10.
6 Arend SM, Cerdá de Palou E, de Haas P, Janssen R, Hoeve MA, Verhard EM, et al. Pneumonia caused by Mycobacterium kansasii in a series of patients without recognised immune defect. Clin Microbiol Infect. 2004 Aug; 10(8):738-48.

7 Sfeir M, Walsh M, Rosa R, Aragon L, Liu SY, Cleary $\mathrm{T}$, et al. Mycobacterium abscessus complex infections: a retrospective cohort study. Open Forum Infect Dis. 2018 Feb; 5(2):ofy022. 
$8 \mathrm{Al}$ Jarad N, Demertzis P, Jones DJ, Barnes NC, Rudd RM, Gaya H, et al. Comparison of characteristics of patients and treatment outcome for pulmonary non-tuberculous mycobacterial infection and pulmonary tuberculosis. Thorax. 1996 Feb;51(2):137-9.

9 Axson EL, Bloom CI, Quint JK. Nontuberculous mycobacterial disease managed within UK primary care, 2006-2016. Eur J Clin Microbiol Infect Dis. 2018 Sep;37(9):1795-803.

10 Al-Harbi A, Al-Jahdali H, Al-Johani S, Baharoon S, Bin Salih S, Khan M. Frequency and clinical significance of respiratory isolates of non-tuberculous mycobacteria in Riyadh, Saudi Arabia. Clin Respir J. 2016 Mar;10(2): 198-203.

11 Varghese B, Enani M, Shoukri M, AlThawadi S, AlJohani S, Al-Hajoj S. Emergence of rare species of nontuberculous mycobacteria as potential pathogens in Saudi Arabian clinical setting. PLoS Negl Trop Dis. 2017 Jan; 11(1):e0005288.

12 Varghese B, Enani M, Shoukri M, AlJohani S, Al Ghafli H, AlThawadi S, et al. The first Saudi Arabian national inventory study revealed the upcoming challenges of highly diverse non-tuberculous mycobacterial diseases. PLoS Negl Trop Dis. 2018 May; 12(5): e0006515.

13 Mokaddas E, Ahmad S. Species spectrum of nontuberculous mycobacteria isolated from clinical specimens in Kuwait. Curr Microbiol. 2008 May;56(5):413-7.

14 Al-Mahruqi SH, van-Ingen J, Al-Busaidy S, Boeree MJ, Al-Zadjali S, Patel A, et al. Clinical relevance of nontuberculous Mycobacteria, Oman. Emerg Infect Dis. 2009 Feb;15(2): 292-4.

15 Mokaddas E, Ahmad S, Samir I. Secular trends in susceptibility patterns of Mycobacterium tuberculosis isolates in Kuwait, 19962005. Int J Tuberc Lung Dis. 2008 Mar;12(3): $319-25$.
16 Ahmad S, Mokaddas E, Al-Mutairi N, Eldeen HS, Mohammadi S. Discordance across phenotypic and molecular methods for drug susceptibility testing of drug-resistant Mycobacterium tuberculosis isolates in a low TB incidence country. PLoS One. 2016 Apr;11(4): e0153563.

17 Ahmad S, Fares E, Araj GF, Chugh TD, Mustafa AS. Prevalence of S315T mutation within the katG gene in isoniazid-resistant clinical Mycobacterium tuberculosis isolates from Dubai and Beirut. Int J Tuberc Lung Dis. 2002 Oct; 6(10):920-6.

18 Mokaddas E, Ahmad S. Development and evaluation of a multiplex PCR for rapid detection and differentiation of Mycobacterium tuberculosis complex members from non-tuberculous mycobacteria. Jpn J Infect Dis. 2007 May;60(2-3):140-4.

19 Al-Mutairi NM, Ahmad S, Mokaddas E. Performance comparison of four methods for detecting multidrug-resistant Mycobacterium tuberculosis strains. Int J Tuberc Lung Dis. 2011 Jan;15(1):110-5.

20 Griffith DE. Nontuberculous mycobacterial lung disease. Curr Opin Infect Dis. 2010 Apr; 23(2):185-90.

21 Lin L, Yin X, Wang Q. Rapid differentiation of mycobacteria by simplex real-time PCR with melting temperature calling analysis. J Appl Microbiol. 2015 Sep;119(3):853-8.

22 Kim JU, Ryu DS, Cha CH, Park SH. Paradigm for diagnosing mycobacterial disease: direct detection and differentiation of Mycobacterium tuberculosis complex and non-tuberculous mycobacteria in clinical specimens using multiplex real-time PCR. J Clin Pathol. 2018 Sep;71(9):774-80.

23 van Eck K, Faro D, Wattenberg M, de Jong A, Kuipers S, van Ingen J. Matrix-assisted laser desorption ionization-time of flight mass spectrometry fails to identify nontuberculous mycobacteria from primary cultures of respiratory samples. J Clin Microbiol. 2016 Jul; 54(7):1915-7.
24 Pranada $A B$, Witt E, Bienia M, Kostrzewa M, Timke M. Accurate differentiation of Mycobacterium chimaera from Mycobacterium intracellulare by MALDI-TOF MS analysis. Med Microbiol. 2017 May;66(5):670-7.

25 Duan H, Han X, Wang Q, Wang J, Wang J, Chu N, et al. Clinical significance of nontuberculous mycobacteria isolated from respiratory specimens in a Chinese tuberculosis tertiary care center. Sci Rep. 2016 Nov;6(1): 36299.

26 Lee H, Han JH, Park HY, Jeon K, Huh HJ, Ki $\mathrm{CS}$, et al. Liquid culture enhances diagnosis of patients with milder forms of non-tuberculous mycobacterial lung disease. Int J Tuberc Lung Dis. 2017 Mar;21(3):345-50.

27 Irandoost M, Zare Ghanbari M, Sakhaee F, Vaziri F, Rahimi Jamnani F, Siadat SD, et al. High rates of Mycobacterium fortuitum isolation in respiratory samples from Iranian patients with suspected tuberculosis: is it clinically important? J Med Microbiol. 2018 Sep; 67(9):1243-8.

28 Koh WJ, Jeong BH, Kim SY, Jeon K, Park KU, Jhun BW, et al. Mycobacterial characteristics and treatment outcomes in Mycobacterium abscessus lung disease. Clin Infect Dis. 2017 Feb;64(3):309-16.

29 Nakanaga K, Sekizuka T, Fukano H, Sakakibara Y, Takeuchi F, Wada S, et al. Discrimination of Mycobacterium abscessus subsp. massiliense from Mycobacterium abscessus subsp. abscessus in clinical isolates by multiplex PCR. J Clin Microbiol. 2014 Jan;52(1):251-9.

30 Wallace RJ Jr, Zhang Y, Wilson RW, Mann L, Rossmoore H. Presence of a single genotype of the newly described species Mycobacterium immunogenum in industrial metalworking fluids associated with hypersensitivity pneumonitis. Appl Environ Microbiol. 2002 Nov;68(11):5580-4. 